\title{
Radio Magnetotelluric (RMT) measurement over lake Mälaren, Sweden: a case study
}

\author{
Suman Mehta \\ Uppsala University, Department of Earth Sciences; Upsala, Sweden; e-mail: suman.mehta@geo.uu.se \\ (c) 2015 Authors. This is an open access publication, which can be used, distributed and reproduced in any medium according \\ to the Creative Commons CC-BY 4.0 License requiring that the original work has been properly cited.
}

Over the past few decades the application of frequency domain radio Magnetotelluric (RMT) have been well established and utilized in the studies of near surface geophysics. This method uses the distance radio transmitter in the frequency range 15 to $250 \mathrm{kHz}$ as source and acts as a tool to model the variation of electrical resistivity of the subsurface. In practice, three component magnetic and two component electric data are measured and recorded. These measured quantities are then used to estimate the Earth's electromagnetic transfer functions called impedance tensor and tipper vector. The transfer function carries the information related to resistivity variation of the subsurface.

The present study was carried out in an urban setting which is a challenging environment for this method. The Swedish Road Authorities (Trafikverket) has proposed and planned several infrastructure projects to cater the immense overload of traffic in the Stockholm city due to growing population. One such project initiated by Swedish road authorities is the Stockholm bypass project that is a ten year construction plan of $21 \mathrm{~km}$ long motor way and to reduce the impact on the environment $18 \mathrm{~km}$ will be located in tunnel. The main objective of this study is thus to identify potential fracture zones and faults as well as the general geological settings. The proposed tunnel will be at a depth of $60 \mathrm{~m}$ and will pass under Lake Mälaren at three areas. This raised an opportunity and challenge to explore the applicability of the land RMT measurement system called EnviroMT (Bastani 2001). This acquisition system developed in Uppsala University has already proved to be a robust tool for land survey. To make it applicable in shallow water environment, an improvisation of existing EnviroMT system was pioneered by Geological Survey of Sweden (SGU). The prototype acquisition system was successfully tested in April 2012 by Geological Survey of Sweden (SGU) on Lake Mälaren. The new system did not only turned out to be appropriate for such a challenging environment, but it was also much more efficient as compared with land surveys. The survey extensively covered all the three water passages. In three days, fifty two lines including 1160 stations with an average spacing of $15 \mathrm{~m}$ were covered.

Urban environment have its own adverse effect on the RMT data. Such cultural noises had to be identified and removed to obtain more accurate and realistic model. Parametric representation combined with a Truncated Singular Value Decomposition (TSVD) regularization described in Bastani \& Pedersen (2001) was used to carry out the processing of the noisy data. Strike analysis of all the profile lines suggested the structure to be mostly $2 \mathrm{D}$ except for some region where multiple strike direction was observed suggesting a 3D structure. Thus the data were inverted to obtain 2D resistivity models using EMILIA (Kalscheuer et al. 2010) for all the lines and 3D models using WSINV3DMT (Siripunvaraporn \& Egbert 2000) for a few selected region. The resistivity models of all the lines had consistent features in term of water depth, sediments thickness and the depth to the bedrock (Mehta et.al 2014). A coherent low resistivity is observed across all the lines and it coincides well with the low velocity zone suggest by 
the available seismic refraction survey in the same region. This is an indication of a possible fracture zone in the bedrock. The comparison of 3D slices with the $2 \mathrm{D}$ resistivity models along the lines further increases the certainty of the fracture zone.

Acknowledgments: Formas, SGU, BeFo, SBUF, Skanska, Boliden, FQM and NGI.

\section{REFERENCES}

Bastani M., 2001. EnviroMT - a new Controlled Source/Radio Magnetotelluric System. Uppsala University [PhD thesis].
Bastani M. \& Pedersen L.B., 2001. Estimation of magnetotelluric transfer functions from radio transmitters. Geophysics, 66, 1038-1051.

Kalscheuer T., García M., Meqbel N. \& Pedersen L.B., 2010. Non-linear model error and resolution properties from two-dimensional single and joint inversions of direct current resistivity and radiomagnetotelluric data. Geophysical Journal International, 182, 3, 1174-1188.

Mehta S., Bastani M., Malhemir A.,Wang S. \& Pederson L., 2014. Shallow water radio-magnetotelluric (RMT) measurements in urban environment: A case study from Stockholm city. EGU General Assembly Conference Abstracts, 16, 4196.

Siripunvaraporn W. \& Egbert G., 2000. An Efficient data-subspace inversion method for $2 \mathrm{D}$ magnetotelluric data. Geophysics, 65, 791-803. 